\title{
VALUE OF DIAGNOSTIC HYSTEROSCOPY IN INFERTILITY AS FIRST LINE INVESTIGATION
}

Vidya Bhat, Prashant S. Joshi, Merlin. J.

1. Consultant Obstetrician \& Gynaecologist, Director Radhakrishna hospital and IVF Centre, Bangalore

2. Associate Professor, Department of OBG, Adichunchanagiri Institute Of Medical Sciences, BG Nagara.

3. Fellowship Resident, Radhakrishna hospital and IVF Centre, Bangalore

\section{CORRESPONDING AUTHOR}

Dr Prashant S. Joshi

Flat no 201, Mannar Sorrento, 36 $6^{\text {th }}$ Main, $3^{\text {rd }}$ Cross, BTM 1 stage, Madivala Dollars Colony, Bangalore

E-mail: joshpj@rediffmail.com

Ph: 00919900865976

\section{ABSTRACT: AIM OF THE STUDY:}

- To evaluate the value of Diagnostic Hysteroscopy in a primary work-up of infertility

- To compare its use in primary versus secondary infertility

MATERIAL \& METHODS: Prospective study from Jan 2012 - Oct 2012 at Radhakrishna Multispecialty Hospital and IVF centre, Girinagar ,Bangalore INCLUSION CRITERIA:

Both primary and secondary infertile women

A Total No. of patients - 170 (110 primary infertility \& 60 secondary infertility) were studied

Intervention Office Hysteroscopy

Main outcome measure Abnormalities of uterine cavity

RESULTS: $30 \%(n=51)$ of the total infertile patients studied had uterine abnormalities $.34 \%$ ( $n=37)$ of primary infertility patients had uterine abnormalities whereas $23 \%(n=14)$ of secondary infertility patients also had uterine abnormalities. The acquired and congenital causes for uterine abnormalities accounted for $20 \%(n=22)$ and $14 \%(n=15)$ respectively in the primary infertility group whereas it accounted for $17 \%(n=10)$ and $6 \%(n=4)$ respectively in the secondary infertility group CONCLUSION: Routine use of diagnostic hysteroscopy in the work-up of infertility is recommended. Diagnostic hysteroscopy has a similar importance in the evaluation of both primary and secondary infertility

\section{KEY WORDS: Hysteroscopy, Infertility}

INTRODUCTION: Infertility related to uterine cavity abnormalities has been estimated to be the etiologic factor in as many as $10 \%-15 \%$ of couples seeking treatment (1). Abnormal intrauterine findings occur in approximately $34 \%-62 \%$ of infertile women $(2,3)$. Because of the high prevalence of uterine abnormalities, evaluation of the uterine cavity is routinely performed in the basic evaluation of infertile women. 
Uterine cavity can be evaluated by hysterosalpingography(HSG),transvaginal ultrasonograpy (TVS ) \& Office Hysteroscopy(OHS).WHO recommends hysteroscopy only when clinical or complementary exams (HSG or TVS) suggests abnormality or after IVF failure. The aim of the present study was to evaluate the value of Diagnostic Hysteroscopy in a primary work-up of infertility and to compare its use in primary versus secondary infertility.

MATERIAL AND METHODS: The subjects were fully informed and signed a study consent form. The subject population consisted of women undergoing infertility investigation at the Radhakrishna Multispecialty Hospital and IVF centre, Girinagar ,Bangalore between January 2012 and October 2012.

Patients were included if 1 ) they were diagnosed as an infertile couple(both primary \& secondary infertility) 2) their age was between 21-44 y, and 3) they were willing to undergo an diagnostic hysteroscopy. Patients were excluded if they 1) had a known or suspected pregnancy, 2) were undergoing active treatment for any sexually transmitted disease or pelvic inflammatory disease.

Patients eligible for study inclusion had a thorough entry history and physical evaluation, as is standard for all infertility patients. All patients were then offered an opportunity for study inclusion and given an oral presentation of study objectives and written copies of the study consent form.

The OHS was performed with a Gynaecare 2-mm rigid office hysteroscope with an additional 1-mm hysteroscopic sheath utilizing a $75^{\circ}$ optical angle via an Striker HD video camera. Distention of the uterine cavity was accomplished with $30-60 \mathrm{ml}$ of saline by manual injection. A single-tooth tenaculum was applied to align the cervical canal and uterine cavity. A study was judged adequate only when the entire uterine cavity and both tubal ostia were visualized during procedure

RESULTS: Hysteroscopy was performed in 170 infertile women: 110 (65\%) were diagnosed with primary infertility and 60 (35\%) with secondary infertility.

The median age in patients with primary infertility was 25 years(range 18 to39) and in secondary infertility it was 32 years ( 22 to 44 ) respectively. In the group with secondary infertility, the parity ranged from 1 to 3 , the spontaneous abortions ranged from 0 to 5 , and induced abortions ranged from 0 to 3 .

$34 \% \quad(n=37)$ of primary infertility patients had uterine abnormalities whereas $23 \%(n=14)$ of secondary infertility patients also had uterine abnormalities. The acquired and congenital causes for uterine abnormalities accounted for $20 \%(\mathrm{n}=22)$ and $14 \%$ $(n=15)$ respectively in the primary infertility group whereas it accounted for $17 \%(n=10)$ and $6 \%$ $(n=4)$ respectively in the secondary infertility group

Endometrial polyp followed by submucous fibroid was the most common acquired intrauterine abnormality in the primary infertility group whereas submucous fibroids was the most common reason for intrauterine abnormality in secondary infertility group. Other abnormalities included cervical stenosis and intrauterine adhesions.

Septate uterus was found in 8 patients of primary infertility group and appeared to be the most common uterine abnormality in our study.

DISCUSSION: Infertility related to uterine factor abnormalities has been estimated to be the etiology in 10\%-15\% of couples seeking treatment (1). Uterine abnormalities are classified as either acquired or congenital in origin. Acquired uterine abnormalities include uterine 
adhesions and uterine fibroids as well as endometrial and cervical polyps. Such uterine abnormalities are poorly vascularized and probably lead to infertility or miscarriages because of their inability to allow proper embryo implantation and growth. Intrauterine adhesions have been implicated in infertility, especially when they fill the uterine cavity as in Asherman's syndrome with amenorrhea (4). Uterine myomas, especially those submucous in location, may predispose one to habitual abortion, and it has been estimated that $41 \%$ of women harboring these lesions may abort (5). Congenital uterine abnormalities consist of fusion anomalies or agenesis of the developing uterus that may include septate, bicornuate, didelphic, or unicornuate uteri. These anomalies occur in one in every 700 women and are believed to cause habitual abortions. It has been shown that the abortion rate in women with the following anomalies is as follows: $34 \%$ with bicornuate uterus, $35 \%$ with a single uterine horn, and $22 \%$ with a septate uterus (6). Bicornuate and unicornuate uteri also lead to unfavorable events later in gestation, including fetal malpresentation and preterm labor. These abnormalities may be treated surgically. Uterine septa can be treated via operative hysteroscopy with improvement in fertility rates. Because of the significant infertility implications caused by such uterine abnormalities, thorough diagnostic and therapeutic procedures are necessitated in the evaluation of infertile women. Abnormal intrauterine findings occur in approximately $34 \%-62 \%$ of infertile women $(2,3)$.The findings of our study were quite similar to other studies like that of Moty Pansky et al(7)

In the current study, $30 \%$ of women, undergoing infertility evaluation, had abnormal uterine findings on hysteroscopy. No significant difference in the rate of uterine pathology was found between women with primary and secondary infertility (34\% and 23\%, respectively). Yet, more cases of endometrial polyp and septate uterus occurred in the group of women with primary infertility. While the relationship between congenital uterine malformations and impaired pregnancy outcome (such as recurrent pregnancy loss, late abortions, preterm deliveries, and malpresentations) is quite established, the issue of these malformations as a cause of infertility is still debatable. The incidence of uterine malformations in other series of infertile patients varies between $1 \%$ and $26 \%$, with a mean incidence of $3.4 \%$. We observed an incidence of uterine malformation for both primary (14\%) and secondary infertility (6\%) . The reported incidence of myomas in women without any obvious cause of infertility is estimated to be between $1 \%$ and $2.4 \%$. In the current study, submucous myomas were diagnosed in $7.05 \%$ of patients with infertility.

Myomas influence fertility. Submucous and intramural myomas distort the cavity, impairing implantation and pregnancy rates in women undergoing IVF. Several theories have been proposed regarding this issue, including alteration of uterine contractility or induction of inflammatory and vascular changes leading to a less receptive implantation site.

In our study endometrial polyps were diagnosed in both primary and secondary infertility groups. The possible role of these polyps in infertility is yet unclear. In the current study, endometrial polyp was the most common acquired uterine abnormality in primary infertility group.

No significant difference was found in the rate of intrauterine adhesions comparing the patients with primary versus secondary infertility. Oliveira(8) also found intrauterine adhesions in $10 \%$ of patients with repeated failed IVF cycles of whom none had undergone previous abortions or other uterine manipulation. 
While debating the need for routine diagnostic hysteroscopy in the evaluation of the infertile woman, one must keep in mind that this procedure today is no longer a complicated "in-patientgeneral-anesthesia one," but rather a simple, fast, outpatient procedure, requiring short training with high success rates.

Diagnostic hysteroscopy allows complete, accurate identification of intrauterine abnormalities that might negatively affect endometrial receptivity and implantation. The information derived from hysteroscopy helps the infertility specialist to institute appropriate therapy, and by doing so improve conception rates over shorter intervals

CONCLUSION: Routine use of diagnostic hysteroscopy in the work-up of infertility is recommended. Diagnostic hysteroscopy has a similar importance in the evaluation of both primary and secondary infertility

\section{REFERENCES:}

1. Wallach EE. The uterine factor in infertility. Fertil Steril 1972;23:138-58.

2. Lindeman H, Mohr J. CO2 hysteroscopy, diagnosis and treatment. Am J Obstet Gynecol 1976;124:129-33.

3. Gallinat A. Hysteroscopy principles and practice. Philadelphia: JBLippincott, 1984.

4. Asherman J. Amenorrhea traumaticum (atretica). J Obstet Gynecol Br Emp 1947;55:2330.

5. Buttram VC Jr., Reiter RC. Uterine leiomyomata: etiology, symptomatology, and management. Fertil Steril 1981;36:433- 45.

6. Jones W. Obstetrical significance of female genital tract anomalies. Obstet Gynecol 1957;10:1039

7. 7.Moty Pansky, Michal Feingold, Ron Sagi, Arie Herman, David Schneider, Reuvit Halperin Diagnostic Hysteroscopy as a Primary Tool in a Basic Infertility Workup JSLS. 2006 Apr-Jun; 10(2): 231-235

8. Oliveira FG, Abdelmassih VG, Diamond MP, Dozortsev D, Nagy ZP, Abdelmassih R. Uterine cavity findings and hysteroscopic interventions in patients undergoing in vitro fertilization-embryo transfer who repeatedly cannot conceive. Fertil Steril.2003;80(6):1371-1375.

Picture.1. Total no. of Patients

\section{Percentage}
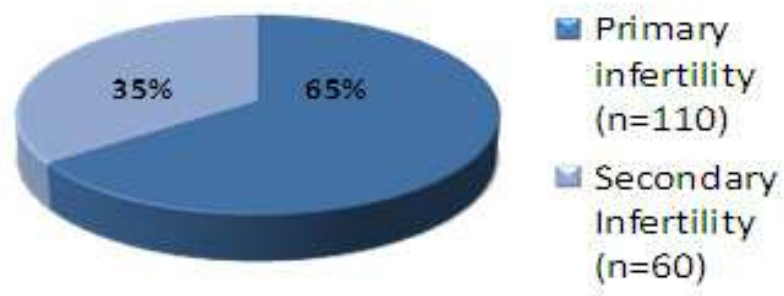
Pictur .2. Age wise distribution of cases

\section{Age (years)}

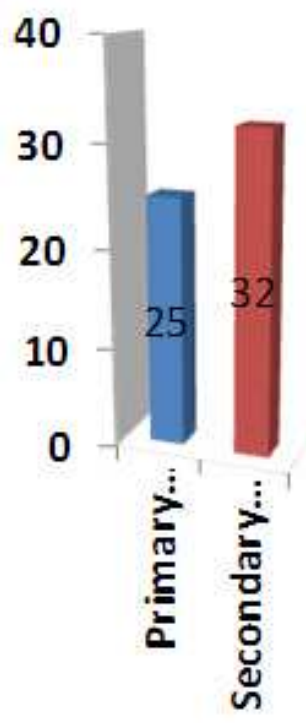

Picture.3.Total no. of intrauterine abnormalities detected

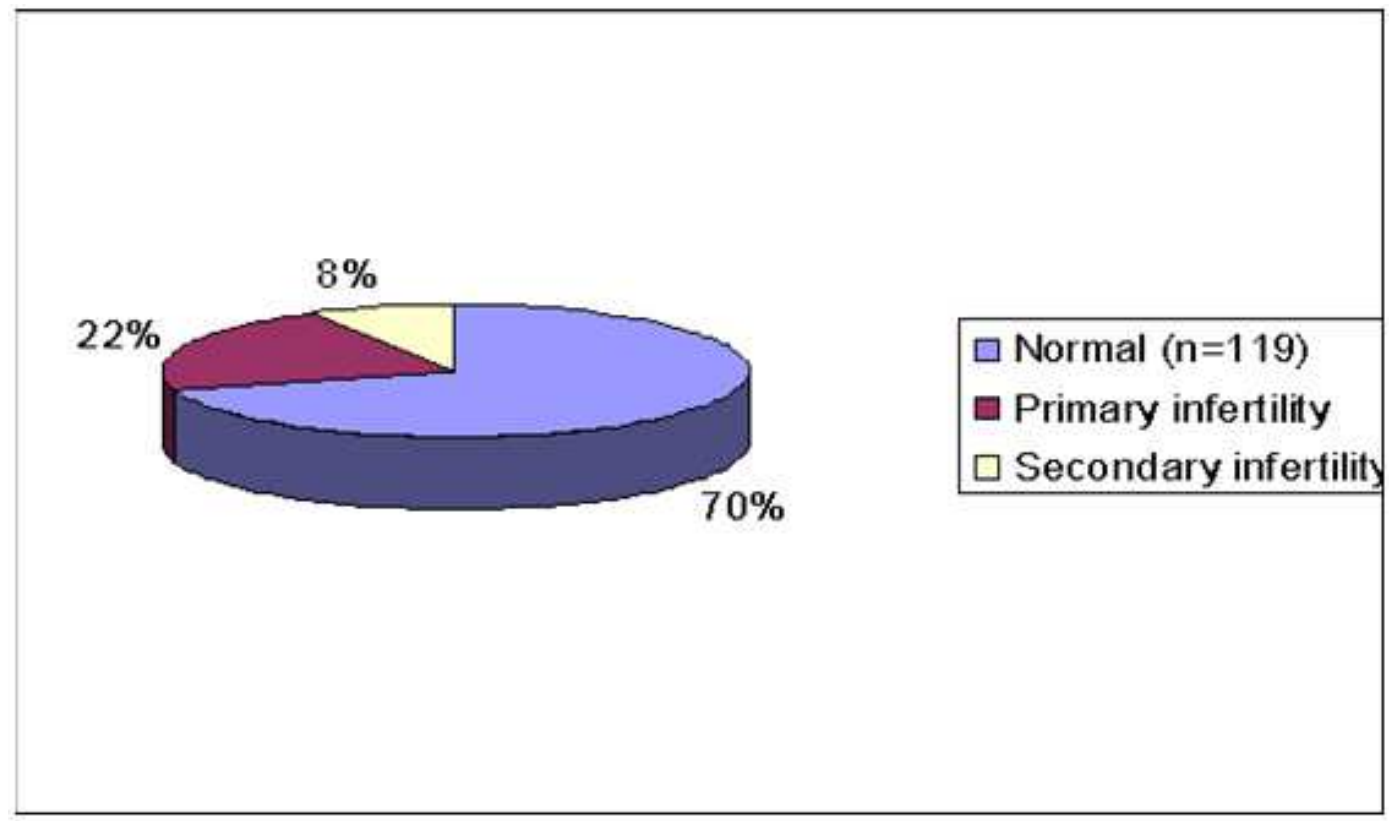


Picture.4. Acquired intra-uterine abnormalities

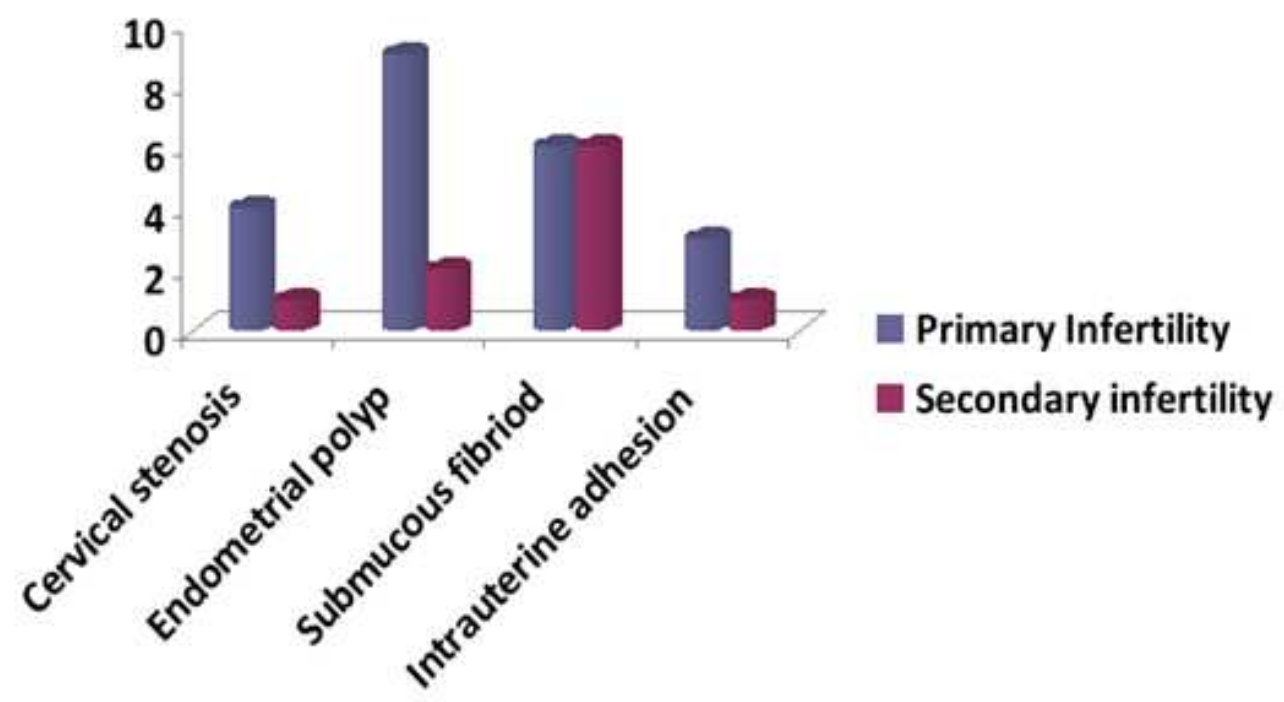

Table.1.

\begin{tabular}{|c|c|c|}
\hline Findings & $\begin{array}{l}\text { Primary infertility } \\
(n)\end{array}$ & Secondary Infertility \\
\hline Normal uterine cavity & $73(66 \%)$ & $46(77 \%)$ \\
\hline Abnormal uterine cavity & $37(34 \%)$ & $14(23 \%)$ \\
\hline Acquired & $22(20 \%)$ & $10(17 \%)$ \\
\hline Congenital & $15(14 \%)$ & $4(6 \%)$ \\
\hline
\end{tabular}

